\title{
Estructura electrónica de aleación AlAs con red zincblende, calculadas con el método LMTO
}

\author{
César Cabrera *1,2 \\ ${ }^{1}$ Universidad Nacional del Callao, Callao, Perú \\ ${ }^{2}$ Universidad Nacional Mayor de San Marcos, Lima, Perú
}

Recibido 02 enero 2020 - Aceptado 25 febrero 2020

\begin{abstract}
Resumen
Se presenta la estructura de bandas de energía, la densidad de estados, la energía prohibida y energía total calculadas con el método de los orbitales lineales muffin-tin (LMTO) en el marco de la teoría del funcional de la densidad (DFT). Se usó un potencial parametrizado en la aproximación de la densidad local (LDA) que usa la aproximación de Barh-Hedin para el término de intercambio y correlación. La estructura electrónica resultó con una brecha indirecta de energía prohíbida de $2.203 \mathrm{eV}$ asociada a la energía total mínima de $-18.10 R y$ que se acomodan bien a los resultados experimentales.
\end{abstract}

Palabras clave: semiconductores, orbitales LMTO, red zincblende.

\section{Electronic structure of AlAs, zinc-blende alloy, calculated with the LMTO method}

\begin{abstract}
The structure of energy bands, the density of states, the prohibited energy and the total energy calculated using the linear muffin-tin orbitals (LMTO) method in the framework of the density functional theory (DFT) in presented. The Schrödinger equation was solved with a parameterized potential in the local density approximation (LDA) with the Barh-Hedin approximation for the term of exchange correlation. The electronic structure resulted in an indirect forbidden energy gap of $2.203 \mathrm{eV}$ associated with a minimum total energy of $-18.10 R y$ and close to the experimental data.
\end{abstract}

Keywords: semiconductors, LMTO orbitals, zincblende lattice.

\section{Introducción}

En las últimas décadas los dispositivos semiconductores han llevado la tecnología moderna de dispositivos a la escala nanométrica, donde los fenomenos cuanticos de carga y de transporte de spin dominan la física del dispositivo. Conocer la estructura electrónica de los semiconductores como el compuesto de Aluminio-Arsénico (AlAs) uno de los materiales electrónicos y optoelectrónicos importantes constituye una ventaja que permite comprender la física a esta escala. En décadas recientes se han reportado estudios sobre las propiedades electrónicas de alta precisión en las que se evidencia el uso de recientes aproximaciones en el término de intercambio y correlación del potencial para el cálculo de estructura electrónica de aleaciones semiconductoras, tales como: cálculo de la estructura de bandas para semiconductores en el marco de la teoría del funcional de la densidad generalizada de Remediakis y Kaxiras (1999) [Rem99], que reporta las bandas de energía del AlAs calculadas empleando la correción para estados exitados propuesta por Fritsche y Gu (1993) [Fri93]; ó el estudio de correcciones a las brechas de banda en teoría del funcional de la densidad de Johnson (1998) [Joh98], que ha reportado la estructura de las bandas de energía del AlAs calculadas con la formulación de GW Hedin's de auto-energía de tipo tight-binding. En ambos estudios se reportó una estructura electrónica de brecha indirecta con banda de conducción de valle mínimo en los puntos de alta simetria $X, \Gamma, L$ en la estructura de bandas.

Estudios mas recientes como la estructura electrónica usando el método kp y los parametros de las bandas de semiconductores de tipo III-V y de sus compuestos de Vurgaftman y Meyer (2001) [Vur01] reportan una estrucutura electrónica del AlAs calculadas con teoría de perturbación de segundo orden; ó el estudio desde primeros

\footnotetext{
*ccabreraa@unmsm.edu.pe
} 
principios de la estructura electrónica de semiconductores tipo zincblende de Wang et al. (2013) [Wan13] que reporta bandas de energía de la aleación de AlAs calculadas usando un potencial de intercambio semilocal propuesto por Tran y Blaha (2009) [Tra09] trabajos en los que se resalta la importancia de la estructura electrónica para el analisis del transporte cuantico en dispositivos semiconductores.

El objetivo es el cálculo de las propiedades electrónicas del AlAs, tales como: la estructura de las bandas de energía, la densidad de estados, la brecha de energía prohibida en el marco de la teoría del funcional de la densidad (DFT) Perdew et al. (2003) [Per96] usando un potencial local en la aproximación de la densidad local (LDA) que depende de la densidad de carga electrónica en las esferas atómicas y de una pequeña fracción de la carga electrónica de valencia transferida a las esferas vacías de la red cristalina, usado con exito para el cálculo de la estructura electrónica de los compuestos de GaP y SiC de Cabrera y Poma (2017) [Cab17] y que resulta una buena alternativa para determinar la estructura electrónica de otras aleaciones semiconductoras.

\section{Fundamento teórico}

De la teoría del funcional de la densidad (DFT) de Kohn (1999) la estructura electrónica de la materia se fundamenta en la ecuación no relativista de Schrödinger para un sistema de muchos electrones con función de onda $\Psi$ y energía $E$, que escrita en unidades atómicas es de la forma [Koh99]:

$\left\{-\frac{1}{2} \sum_{j} \nabla_{j}^{2}-\sum_{j, l} \frac{Z_{l}}{\left|r_{j}-R_{l}\right|}+\frac{1}{2} \sum_{j \neq j^{\prime}} \frac{1}{\left|r_{j}-r_{j^{\prime}}\right|}-E\right\} \Psi=0$

que de acuerdo con el principio variacional de HohenbergKohn (1964), $E$ es de valor mínimo para una correcta densidad de carga electrónica $n(r)$ del estado fundamental [Hoh64]. Densidad de carga electrónica que depende de la función de onda $\psi_{j}$ de un electrón, definida como:

$$
n(r)=\sum_{j=1}^{N}\left|\psi_{j}\right|^{2}
$$

Despues de aplicar formalmente el cálculo de valores extremos a la ecuación (1), lleva el problema de la ecuación de Schrödinger para un sistema de muchos electrones y que no se detallará aqui, al problema de resolver la ecuación de Schrödinger auto-consistente de un solo electrón que se mueve en un campo de potencial efectivo, que escrita en unidades atómicas es de la forma:

$$
\left\{\frac{1}{2} \nabla^{2}+V_{e f}(r)-\epsilon_{i}\right\} \psi_{i}(r)=0
$$

Un electrón que interacciona con un potencial efectivo $V_{e f f}(r)$ que depende las propiedades cristalinas y de la densidad de carga electrónica $n(r)$ del sólido cristalino en estudio. Para estructuras cristalinas abiertas como la red zincblende de AlAs, este potencial efectivo depende adicionalmente de la pequeña fracción de la carga electrónica que reside en las esferas vacías de la red y que en unidades atomicas es:

$$
V_{e f}(r)=v(r)+\frac{1}{2} \int \frac{n\left(r^{\prime}\right)}{\left|r-r^{\prime}\right|} d r^{\prime}+V_{x c}(r)
$$

En la que el último término $V_{x c}(r)$ es el potencial de intercambio y correlación con dependencia funcional de la densidad $n(r)$ no conocida y se suele tratar con aproximaciones. Tales como los potenciales de intercambio y correlación de densidad de spin local parametrizada reportado por McLaren (1991) [McL91]. Aqui el potencial efectivo se calculó con la aproximación de VonBarhHedin (1972), la aproximación del potencial de intercambio y correlación para un gas homogeneo de electrones de spin polarizado [Von72].

Este conjunto de ecuaciones son conocidas como las ecuaciones auto-consistentes de Kohn-Sham (KS) para el cálculo de la estructura electrónica. Por otro lado la energía del estado fundamental del sistema esta dada por:

$$
\begin{aligned}
E= & \sum_{j} \epsilon_{j}+E_{x c}[n(r)]-\int V_{x c}(r) n(r) d r^{3} \\
& -\frac{1}{2} \int \frac{n(r) n\left(r^{\prime}\right)}{\left|r-r^{\prime}\right|} d r^{3}
\end{aligned}
$$

Ecuación que se puede calcular, puesto que resuelta la ecuación de Schrödinger, ya se conoce los auto-valores de energía $\epsilon_{i}$ y las funciones de onda. El término $E_{x c}(n(r))$ es la energía potencial de intercambio y correlación, también con dependencia funcional de la densidad de carga electrónica y tratada con la aproximación de VonBarhHedin.

\begin{tabular}{lcccr}
\hline$a(\mathrm{~A})$ & $R_{A l}(\mathrm{au})$ & $R_{A s}(\mathrm{au})$ & $R_{3}(\mathrm{au})$ & $R_{4}(\mathrm{au})$ \\
\hline 5.62 & 2.522 & 2.745 & 2.522 & 2.745 \\
\hline
\end{tabular}

Tabla 1: Constante de la red y radio de las esferas atómicas y vacías en la red cristalina del AlAs.

\section{Datos}

El compuesto de Aluminio-Arsénico (AlAs) en su estado fundamental tiene estructura cristalina cúbica tipo blenda de cinc (zincblende) con átomos diferente clase. La red cristalina fue llenada con esferas atómicas y vacías de diferente tamaño, de modo que el radio $R_{w s}$ de las esferas se determinaron preservando la simetria y la densidad del material. En la tabla 1. se muestra el parámetro de la red cristalina, Ashcroft y Mermin (1976) y el radio de las cuatro esferas que ocupan el volumen de 
la celda unitaria, necesarios para el cálculo del potencial efectivo $V_{e f}(r)$ que será usado para resolver la ecuación de Schrödinger del sólido [Ash76].

\section{Metodología}

El cálculo de la estructura electrónica y de la energía total fué realizado por etapas. Primera etapa: El cálculo del potencial efectivo de las esferas atómicas y vacías de la red cristalina del AlAs. Potencial con dependencia funcional de la densidad de carga electrónica $\rho(r)$ de los átomos en la red cristalina y de una fracción pequeña de la carga electrónica $q_{R}$ que reside en las esferas vacías de la red. Este potencial para la red cristalina zincblende y en la aproximación de las esferas atómicas ASA, Skriver (1984) [Skr84], es:

$$
\begin{aligned}
V_{e f}(r)= & -\frac{2 Z}{r}+\frac{2}{r} \int_{0}^{r} 4 \pi\left(r^{\prime}\right)^{2} \rho\left(r^{\prime}\right) d r^{\prime} \\
& +2 \int_{r}^{R_{w s}} \frac{4 \pi\left(r^{\prime}\right)^{2} \rho\left(r^{\prime}\right)}{r^{\prime}} d r^{\prime}+2 V_{x c}(r) \\
& -\sum_{\overrightarrow{R^{\prime}}} \sum_{\vec{T}}^{4} \frac{2 q_{\overrightarrow{R^{\prime}}}}{\left|\vec{R}-\overrightarrow{R^{\prime}}-\vec{T}\right|}
\end{aligned}
$$

Para la cantidad $V_{x c}(r)$ se usó la aproximación de VonBarth-Hedin (1972) [Von72], el potencial de intercambio y correlación para un gas homogeneo de electrones de spin polarizado. El último término es la contribución al potencial efectivo, de la fracción de la carga $q_{R}$ que reside en las esferas vacías de la red. $\vec{R}$ denota la posición de la esfera vacía en el centro de la diagonal, $\overrightarrow{R^{\prime}}$ la posición de la esfera vacía situada a $3 / 4$ de la diagonal y $\vec{T}$ la posición de las otras esferas vacías de la red. Este potencial se calculó con una densidad de carga electrónica similar a la del átomo libre dentro de las esferas atómicas y una pequeña fracción de la carga residiendo homogeneamente en las esferas vacías.

Segunda etapa: La ecuación de Schrödinger para el sólido cristalino fue resuelta con este potencial efectivo $V_{e f}(r)$ y el método de los orbitales LMTO, la ecuación de Schrödinger se transformó en el problema del cálculo de los auto-valores. Estos auto-valores de energía $\epsilon_{i}$ son calculadas en el espacio recíproco y en las direcciones cristalográficas de alta simetría que toma el vector de onda $\vec{k}$, lo que constituye la estructura de las bandas de energía y a la vez permitió calcular la densidad de estados ocupados (DOS) del sólido cristalino.

Tercera etapa: La energía total fue determinada calculando las integrales de la ecuación (5) usando la densidad de carga electrónica $\rho(r)$ de la red cristalina y la densidad de estados DOS, que ya fueron calculadas cuando se resolvió la ecuación de Schrödinger del sólido.

\section{Resultados y discusión}

La relación de dispersión $\epsilon=\epsilon(k)$, que tecnicamente constituye la estructura de las bandas de energía fue calculada para diferentes porcentajes de la pequeña fracción de la carga electrónica que reside en las esferas vacías. La estructura de las bandas de energía calculadas para $\gamma=0.0$ es presentada en la Figura 1, corresponde a toda la pequeña fracción de la carga $q_{R}$ en las esferas vacías. La estructura de las bandas de energía se obtuvo con una brecha indirecta de energía prohibida, entre el tope de la banda de valencia ubicada en el punto $\Gamma_{25} \mathrm{y}$ el fondo de la banda de conducción ubicada en el punto $X_{5}$. El perfil de la estructura de las bandas de energía es similar a lo reportado por Vurgaftman y Meyer (2001) con otro método.

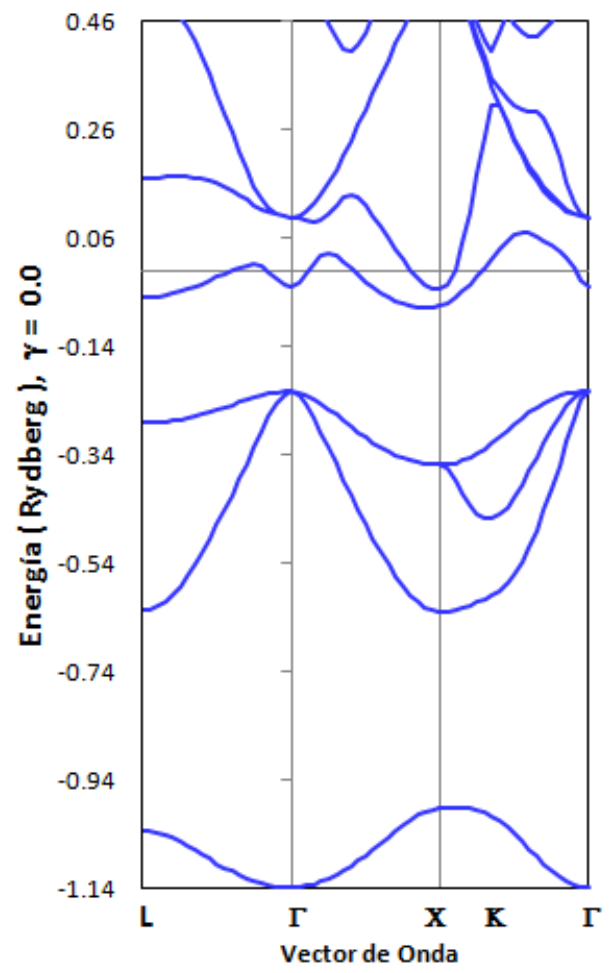

Figura 1: Bandas de energía del AlAs obtenida para $\gamma=0.0$, toda la pequeña carga electrónica $q_{R}$ residiendo en las esferas vacias de la red.

La estructura de las bandas de energía obtenidas para $\gamma=1.0$, que corresponde al caso de esferas vacías sin nada de la pequeña fracción de la carga electrónica $q_{R}$, es mostrada en la Figura 2. Aquí el perfil de la estructura de bandas también presenta una brecha indirecta de energía prohibida, pero de menor magnitud.

A continuación presentamos los valores de energía de las principales direcciones de simetría cristalográfica de 
la red cristalina. Estas fueron obtenidas de la estructura de las bandas de energía calculadas para $\gamma=0.0$, asociada a la mínima energía total del sistema calculada en el presente trabajo.

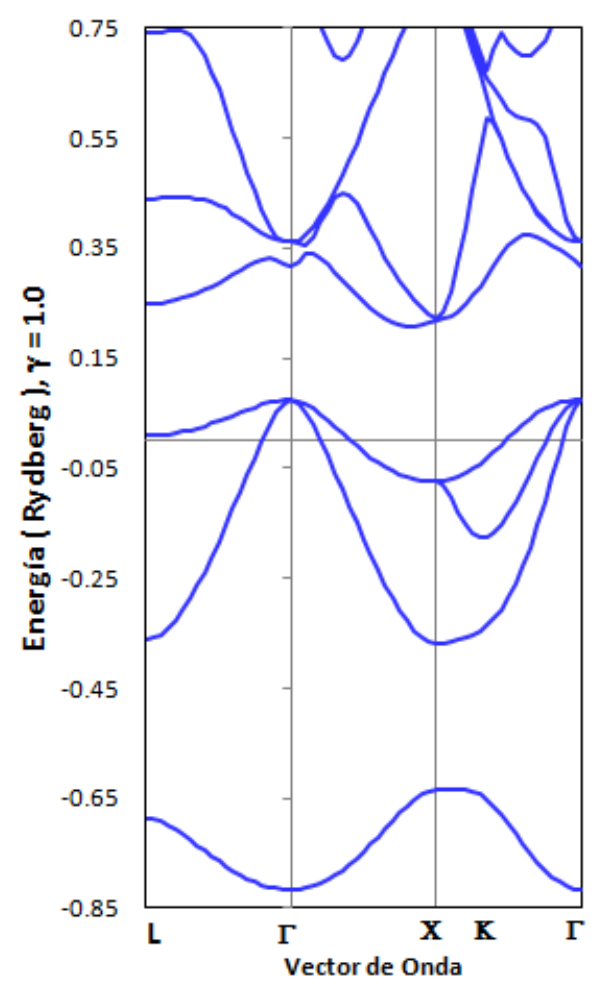

Figura 2: Bandas de energía del AlAs obtenidas para $\gamma=1.0$, sin carga electrónica residiendo en las esferas vacías de la red.

\begin{tabular}{lcccr}
\hline$P S$ & $E_{g}(\mathrm{Ry})$ & $E_{g}(\mathrm{eV})$ & $E_{L}(\mathrm{eV})$ & $E_{e}(\mathrm{eV})$ \\
\hline$\Gamma$ & 0.194 & 2.64 & 3.087 & 3.099 \\
$X$ & 0.162 & 2.20 & 2.240 & 2.240 \\
$L$ & 0.174 & 2.36 & 2.800 & 2.460 \\
\hline
\end{tabular}

Tabla 2: Brecha de energía $E_{g}$ de los mínimos de la banda de conducción en los puntos de simetria $\Gamma, X$ y $L$ con respecto del máximo de la banda de valencia en el punto $\Gamma$, obtenidas de las bandas de energía en este trabajo, $E_{L}$ obtenida por Wang (2013) [Wan13], $E_{e}$ valor experimetal Vurgaftman y Meyer (2001) [Vur01].

La densidad de estados (DOS) calculada para $\gamma=0.0$, que corresponde a toda la pequeña fracción de la carga electrónica $q_{R}$ residiendo en las esferas vacías, es mostrada en la Figura 3. El perfil de esta DOS confirma la brecha de la energía prohibida obtenida en la estructura de las bandas de energía para el caso de $\gamma=0.0$

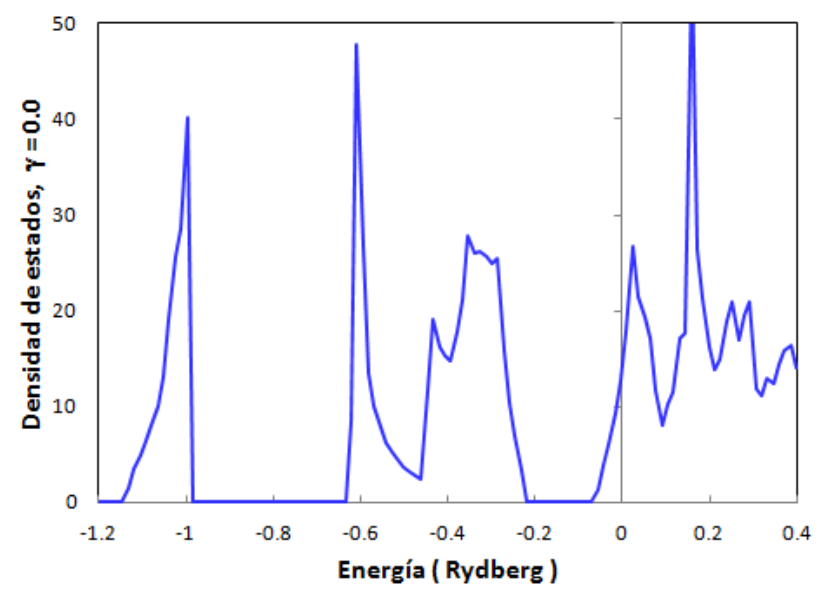

Figura 3: Densidad de estados del AlAS obtenidas para $\gamma=0.0$, toda la pequeña carga electrónica $q_{R}$ residiendo en las esferas vacias de la red.

La densidad de estados DOS obtenida para $\gamma=1.0$, caso en que nada de la pequeña fraccion de la carga electrónica $q_{R}$ reside en las esferas vacías de la red cristalina, es mostrada en la Figura 4. Este perfil de la DOS presenta un desplazamiento hacia mayores valores de energía y presenta la brecha de energía prohibida obtenida en los calculos de la estructura de las bandas de energía calculadas para el caso de $\gamma=1.0$

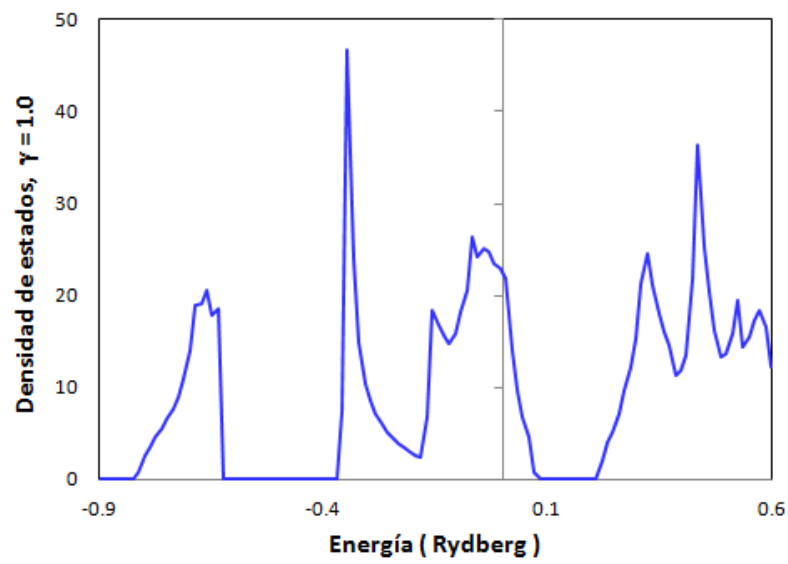

Figura 4: Densidad de estados del AlAs obtenidas para $\gamma=1.0$, con nada de la pequeña carga electrónica residiendo en las esferas vacías de la red.

La energía prohibida, brecha entre el fondo de la banda de conducción y el tope de la banda de valencia, obtenida de la estructura de las bandas de energía, así como la energía total, fueron calculadas para diferente 
porcentaje de la pequeña fracción de la carga electrónica $q_{R}$ residiendo en las esferas vacías de la red cristalina. La Tabla 3 nos muestra los resultados obtenidos.

\begin{tabular}{lccr}
\hline$\gamma$ & $E_{g}(\mathrm{eV})$ & $E_{F}(\mathrm{Ry})$ & $E_{T}(\mathrm{Ry})$ \\
\hline 0.0 & 2.203 & -0.218 & -18.10 \\
0.2 & 1.463 & -0.165 & -18.04 \\
0.4 & 1.098 & -0.118 & -17.76 \\
0.6 & 0.548 & -0.065 & -17.81 \\
0.8 & 0.171 & -0.004 & -17.86 \\
1.0 & 1.714 & -0.083 & -17.54 \\
\hline
\end{tabular}

Tabla 3: Brecha de energía prohibida $E_{g}$, energía de Fermi $E_{F}$ y la energía total $E_{T}$ del AlAs, calculadas para diferentes valores de la pequeña fracción de la carga electrónica $q_{R}$ residiendo en las esferas vacías de la red cristalina.

Como consta en la tabla 3., la máxima brecha de energía prohibida de $2.203 \mathrm{eV}$ obtenida para $\gamma=0.0$, que difiere de la energía prohibida $2.573 \mathrm{eV}$ reportado Remediakis y Kaxiras (1999) ó del valor de la energía prohibida $2.18 \mathrm{eV}$ reportada por Godby y Sham (1986) [God86] y cercana de la energía prohibida de 2.24 eV reportada por Vurgaftman y Meyer (2001).

\section{Energía Prohibida de AIAs}

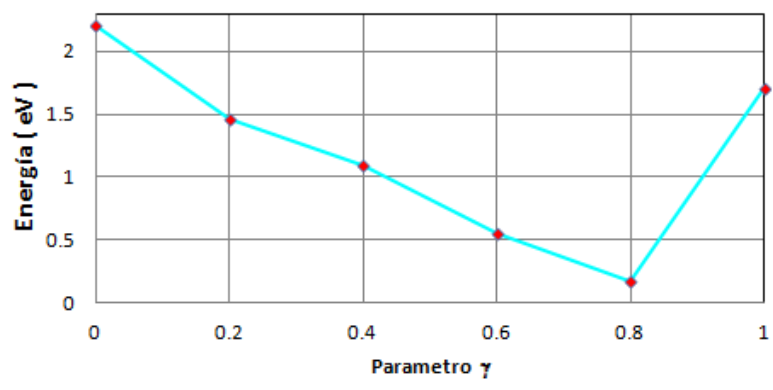

Figura 5: Brecha de energía prohibida del AlAs (en eV), calculadas para diferentes valores de la carga electronica $q_{R}$ residiendo en las esferas vacías de la red.

La energía total mínima resultó de $-18.10 R y$ obtenida para $\gamma=0.0$, energía mínima asociada a la estructura de las bandas de energía con la brecha de máximo valor.

\section{Energía Total de AIAs}

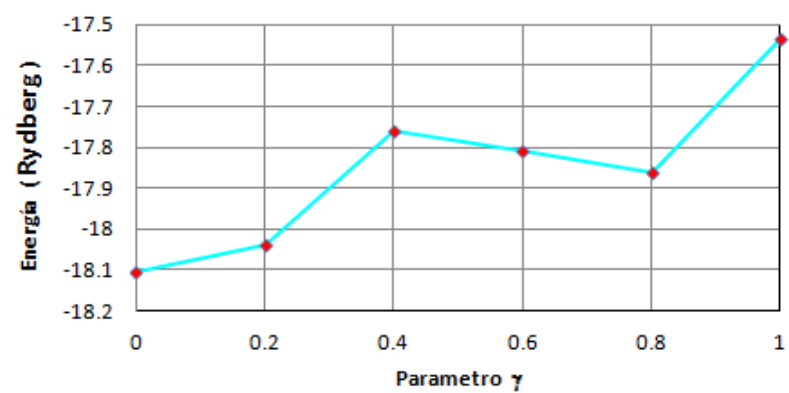

Figura 6: Energía Total del AlAs (en Ry), calculadas para diferentes valores de la carga electronica $q_{R}$ residiendo en las esferas vacías de la red.

\section{Conclusiones}

La mejor estructura electrónica calculada para esta aleación de AlAs es la obtenida para el caso de toda la pequeña fracción de la carga electrónica $q_{R}$ residiendo en las esferas vacías de la red cristalina. La estructura de las bandas de energía con brecha indirecta de energía prohibida de $2.203 \mathrm{eV}$, brecha de energía confirmada en el perfil de la densidad de estados DOS calculada para el mismo caso de $\gamma=0.0$, se ajustan muy bien a la energía prohibida obtenidas con otros métodos como: al valor $2.18 \mathrm{eV}$ obtenido por Johnson y Ashcroft (1998) [Joh98]; al valor $2.573 \mathrm{eV}$ obtenido por Remediakis y Kaxiras (1999) [Rem99] y al valor de $2.24 \mathrm{eV}$ reportado por Vurgaftman y Meyer (2001) [Vur01].

Los resultados muestran que el caso de toda la pequeña fracción de la carga electrónica residiendo en las esferas vacías de la red cristalina, es la que minimiza la energía total de la alaeción de AlAs. Con una energía total de $-18.10 R y$ obtenida para $\gamma=0.0$ implicando una mayor estabilidad del sistema. Lo que permite concluir que el método LMTO y la teoría del funcional de la densidad, Perdew et al. (1996), son buenos para el estudio teórico de la estructura electrónica de materiales y aleaciones [Per96].

\section{Agradecimientos}

Sinceros agradecimientos a la Facultad de Ciencias Físicas de la UNMSM y a la FIQ-UNAC.

\section{Referencias}

[Ash76] Ashcroft, N. and Mermin, N. (1976). Solid State Physics, New York, USA: Holt Rinehart and Wis- ton.

[Cab17] Cabrera, C. y Poma, M. (2017). Estructura electrónica y energía total de los sistemas cristalinos de 
Ge, GaP y $S i C$, Revista de investigación de Física. vol 20, 2, pp 1-4.

[Fri93] Fritsche, L. and, Gu, M. (1993) Binding properties of $3 d$ transicion metals in generalized density functional theory Phys. Rev. B. 48, 4250.

[God86] Godby, R. and, Sham, L. (1986) Accurate exchange-correlation potential for silicon and its discontinuity on addition of an electron Phys. Rev. Lett. 56, 2415.

[Hoh64] Hohemberg, P. and Kohn, W. (1964) Inhomogeneous electron gas Physics Review B. 136, 864.

[Joh98] Johnson, K. and Ashcroft, N. (1998). Corrections to density functional theory band gaps Physical review B, 23, vol. 58, pp 15548-15556.

[Koh99] Kohn, W. (1999). Electronic structure of matter wave functions and density functionals Rev. Mod. Phys. 5, vol. 71, 1253-1266.

[McL91] McLaren, J. (1991). Parameterised local spin density exchange-correlation energies and potentials for electronic structure calculations Comp. Phys. Comms. 66, pp 383-391.
[Per96] Perdew, J., Burke, K. and Eenzerhof, M. (1996). Generalized Gradient Approximation Made Simple. Phys. Rev. Lett. 77, pp 3865.

[Rem99] Remediakis, I. and Kaxiras, E. (1999). Band structure calculation for semiconductors within generalized density functional theory, Physical Review B, Vol. 59, 8, pp 5536-5543.

[Skr84] Skriver, H. (1984). The LMTO Method: MuffiTin Orbitals and Electronic Structure Berlin, Germany, Springer-Verlag.

[Tra09] Tran, F. and Blaha, P. (2009); Physical Review Letters. 102, pp 226401.

[Vur01] Vurgaftman, I. and Meyer, J. (2001). Band parameters III- $V$ semiconductors and their alloys Physical Review B, 11, vol. 89, pp 5815-5876.

[Von72] Von Barth, U. and Hedin, L. (1972). Exchange correlations energy Journal Physics C. 5, pp 1629.

[Wan13] Wang, Y., Haitao, Y., Cao, R., Zahid, F. and Guo, H. (2013). Electronic structure of III-V zincblende semiconductors from first principles, Physical Review B, Vol. 23, 87, pp 235203-1 235203-5. 